\title{
Iron line afterglows: General constraints
}

\author{
G. Ghisellini ${ }^{1}$, D. Lazzati ${ }^{1,2}$, and S. Campana ${ }^{1}$ \\ 1 Osservatorio Astronomico di Brera, Via Bianchi 46, I-23807 Merate (Lc), Italy \\ 2 Dipartimento di Fisica, Università degli Studi di Milano, Via Celoria 16, I-20133 Milano, Italy
}

Received December 18, 1998; accepted March 26, 1999

\begin{abstract}
The discovery of a powerful and transient iron line feature in the X-ray afterglow spectra of $\gamma$-ray bursts would be a major breakthrough for understanding the nature of their progenitors, strongly suggesting the presence of a large, iron rich, mass in the vicinity of the burst event. Model-independent limits to the size and the mass of the the iron line emitting region are derived and discussed. We also discuss how these results can be used to constrain the amount of beaming or anisotropy of the burst emission.
\end{abstract}

Key words: gamma-rays: bursts — supernovae: general — $\mathrm{X}$-rays: general

\section{Introduction}

At this meeting it has been reported the possible detection of an iron line in the $\mathrm{X}$-ray afterglow spectra of two bursts: GRB 970508 detected by BeppoSAX (Piro et al. 1999, see also these proceedings) and GRB 970828 by ASCA (Yoshida et al. these proceedings). The significance of these features is admittedly not extremely compelling ( $\sim 99 \%$ and $\sim 98 \%$ for the two events, respectively), but the implications that they bear are so important to justify a study on the radiation mechanisms that would produce it (Lazzati et al. 1999, see also these proceedings). We can also put strong constraints on the size and mass of the line emitting region in a model-independent way. These limits, which are very robust, point towards the presence, within a distance of $\sim 10^{16} \mathrm{~cm}$ from the burst, of at least $10^{-3} M_{\odot}$ of iron. We also stress that these emission line features, observed in bursts which also have an optical afterglow, strongly suggest that the line emitting region is not spherically symmetric, but must have some degree of anisotropy. Finally, this constrains the possible anisotropy of the burst radiation, since the line emitting region samples different line of sights than our own.

The cosmological parameters will be set throughout this paper to $H_{0}=65 \mathrm{~km} \mathrm{~s}^{-1} \mathrm{Mpc}^{-1}, q_{0}=0.5$ and $\Lambda=0$.

\section{General constraints}

We here prefer to discuss the implications of detecting an iron line during the $\mathrm{X}$-ray afterglow of a generic burst, without referring in particular to the two cases mentioned in the introduction. Let us therefore assume that the flux of the $\mathrm{X}$-ray afterglow is of the order of $10^{-13} \mathrm{erg} \mathrm{cm}^{-2} \mathrm{~s}^{-1}$, and assume a redshift of $z=1$. To be visible during the $\mathrm{X}$-ray afterglow emission, the emission line should have a comparable flux ${ }^{1}, F_{\mathrm{Fe}}=$ $10^{-13} \mathrm{~F}_{\mathrm{Fe},-13} \mathrm{erg} \mathrm{cm}^{-2} \mathrm{~s}^{-1}$. This in itself constrains both the amount of line-emitting matter and the size of the emitting region.

\subsection{Limit to the size}

Assume for simplicity that the emitting region is a homogeneous spherical shell at a distance $R$ from the $\gamma$-ray burst, with a width $\Delta R \leq R$. The fluence of the emission line cannot exceed the absorbed ionizing fluence $q \mathcal{F}$ (where $\mathcal{F}$ is the total GRB fluence and $q$ is the fraction of it which is absorbed and reprocessed into the line). The observed duration of the emission line cannot be shorter than the light crossing time of the region $R / c$. From this we obtain the limit

$R<310^{18} \frac{q \mathcal{F}_{-5}}{F_{\mathrm{Fe},-13}} \quad \mathrm{~cm}$

Since $q$ is at most $\sim 0.03$, the emitting region is very compact, ruling out emission from interstellar matter, even assuming the large densities appropriate for star forming regions. We would like to stress that the above limit is independent of the variability of the line flux.

\subsection{Limit to the mass}

The total line photons produced at $6.4-6.9 \mathrm{keV}$ in $10^{5} t_{5}$ seconds, for a GRB located at $z=1$, are $\sim$

\footnotetext{
${ }^{1}$ Here and in the following we parametrize a quantity $Q$ as $Q=10^{x} Q_{x}$ and adopt cgs units.
} 
$310^{57} F_{\mathrm{Fe},-13} t_{5}$. Assuming that each iron atom produces $k$ line photons, this corresponds to $\sim 150 F_{\mathrm{Fe},-13} t_{5} / k M_{\odot}$ of iron. The parameter $k$ depends on the details of the assumed scenario, but we can set some general limits. Assume in fact that each iron atom can emit photons only when illuminated by an ionizing flux, which is provided by the burst itself or by the high energy tail of the afterglow emission. Since the burst radiation has enough power to photoionize all the matter in the vicinity of the progenitor (see e.g. Boettcher et al. 1999), line photons will be emitted only through the recombination process. But even if we assume that the recombination is instantaneous, the value of the parameter $k$ will not be larger than the total number of photoionizations an ion can undergo during the burst and/or the afterglow. For iron $K$-shell electrons, with cross section $\sigma_{K}=1.210^{-20} \mathrm{~cm}^{2}$ we have:

$k \lesssim \frac{q E}{4 \pi \epsilon_{\text {ion }} R^{2}} \sigma_{K}=6.510^{6} \frac{q E_{52}}{R_{16}^{2}}\left(\frac{9.1 \mathrm{keV}}{\epsilon_{\text {ion }}}\right)$

where $E$ is the total energy emitted by the burst and/or afterglow and $\epsilon_{\text {ion }}$ the energy of a single ionizing photon. This upper limit on $k$ translates in a lower limit on the iron mass:

$M_{\mathrm{Fe}} \gtrsim 2.310^{-5} F_{\mathrm{Fe},-13} t_{5} \frac{R_{16}^{2}}{q E_{52}} M_{\odot}$

which, for a solar iron abundance, yields a total mass $M \gtrsim 0.013 F_{\mathrm{Fe},-13} t_{5} R_{16}^{2} /\left(q E_{52}\right) M_{\odot}$, i.e. a third of a solar mass for $q \sim 0.03$.

Even if these numbers apparently do not rule out reverberation from a molecular cloud (Ghisellini et al. 1999; Mészáros \& Ress 1998), we remark that the recombination time has been assumed negligible in the above discussion. At densities typical of molecular clouds the recombination time is larger than the burst duration and the value of $k$ cannot exceed 12, set by the photoelectric yield of the iron atom (e.g. Boettcher et al. 1999).

\subsection{Limit on geometry and isotropy}

Optical afterglow emission has been observed in about half the $\gamma$-ray burst events for which the X-ray afterglow has been detected. In particular, the optical afterglow of GRB 970508 lasted for hundreds of days (Galama et al. 1998). If the iron line emitting region were spherically symmetric, it would inevitably stop the fireball and the usual relatively slow transformation of bulk kinetic energy into radiation could not take place. For this reason it is necessary to assume some special geometry of the iron line emitting material, which has not to interfere with the observed optical afterglow. In other words, this region cannot be located along our line of sight, but, on the other hand, it has to be illuminated by the burst emission, in order to produce the iron line feature (e.g. a torus surrounding the central region, or a bicone). Therefore we conclude that the iron line feature is a powerful tool to know how isotropic the burst emission is.

\section{Discussion}

We have derived some model-independent limits on the size and mass of the material responsible for iron line features, assuming that they can be detected during the X-ray afterglow. This work has been stimulated by the recent claim of detection of such lines, but the validity of our conclusions are quite general. The most important implication of these lines, if confirmed, regard the progenitor of the burst. In fact it is inescapable to assume that about a solar mass of (probably iron rich) material is located in the close vicinity of the event. Among the proposed models, the Supranova of Vietri \& Stella (1998) is the only one which naturally accounts for this. This in turn implies that at least a class of $\gamma$-ray burst have to be associated with supernovae exploded about a month earlier (assuming that the remnants have a velocity of about $10^{4} \mathrm{~km} \mathrm{~s}^{-1}$ ).

The possible association of GRB with supernovae has been investigated recently in detail by Bloom et al. (1998), Kippen et al. (1998) and Wang \& Wheeler (1998), following the explosion of GRB 980425, likely associated with the type Ic SN 1998bw. Among these works, only Wang \& Wheeler (1998) find evidence for a connection while the other two limit to a few percent the bursts possibly associated with supernovae. In the Supranova scenario, however, the association of supernovae with bursts suffer for a time delay which would smear the time correlation between the two phenomena.

Should the iron lines possibly detected in GRB 970508 and GRB 970828 be real and confirmed by other cases, then we have a strong case for the connection between supernovae and $\gamma$-ray bursts. The next generation of experiments and satellites, such as XMM, AXAF and ASTROE, will provide us with the necessary information to draw more accurate conclusion on the puzzling problem of the $\gamma$-ray burst progenitor.

\section{References}

Bloom J.S., Kulkarni S.R., Harrison F., Prince T., Phinney E.S., 1998, ApJ 506, L105

Boettcher M., Dermer C.D., Crider A.W., Liang E.P., 1999, A\&A 343, 111

Galama T.J., et al., 1998, ApJ 497, L13

Ghisellini G., Haardt F., Campana S., Lazzati D., Covino S., 1999, ApJ (in press) (astro-ph/9808156)

Lazzati D., Campana S., Ghisellini G., 1999, MNRAS 304, L31

Kippen R.M., et al., 1998, ApJ 506, L27

Mészáros P., Rees M.J., 1998, MNRAS 299, L10

Piro L., et al., 1999, 514, L73

Vietri M., Stella L., 1998, ApJ 507, L45

Wang L., Wheeler J.C., 1998, ApJ 504, L87 